\title{
MEASUREMENT AND MODELLING OF GRADIENT MAGNETIC FIELDS FOR BIO-CHEMICAL SEPARATION PROCESSES
}

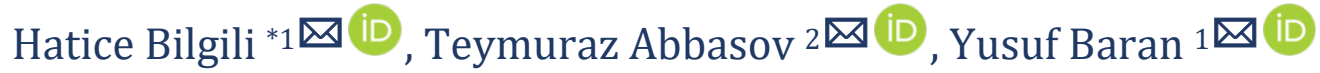 \\ *1 Department of Biomedical Engineering, Inonu University, 44280 Malatya, Turkey \\ 2 Department of Electrical and Electronics Engineering, Inonu University, 44280 Malatya, Turkey
}

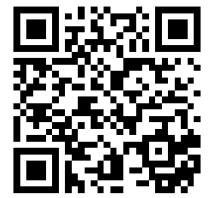

DOI: https://doi.org/10.29121/IJOEST.v5.i2.2021.174

Article Type: Research Article

Article Citation: Hatice Bilgili, Teymuraz Abbasov, and Yusuf Baran. (2021). MEASUREMENT AND

MODELLING OF GRADIENT

MAGNETIC FIELDS FOR BIO-

CHEMICAL SEPARATION

PROCESSES. International Journal of Engineering Science Technologies, $5(2), 69-80$.

https://doi.org/10.29121/IJOEST.v

5.i2.2021.174

Received Date: 15 March 2021

Accepted Date: 10 April 2021

Keywords:

Magnetic Field Intensity

Magnetic Field Gradient

Biomagnetic Separation

NdFeB Magnet

Magnetic Drug Targeting

\begin{abstract}
Separation processes are widely used in chemical and biotechnical processes. Especially biomagnetic separation is an important issue among effective separation processes to separate the magnetic micron and submicron particles. It is necessary to establish and determine a high magnetic field or field gradient in the separation cell. However, it is not easy to determine the magnetic field gradient in the working region for different separation in practice. The reason for these difficulties is that the magnetic cells used in biochemical separation have different geometries and there are no simple and useful systems to easily measure these magnetic fields. Two main objectives are aimed in this study. First, a simple measuring device design can measure gradient magnetic fields with high precision of about $0,01 \mathrm{~mm}$ and, secondly, obtain simple empirical expressions for the magnetic field. A magnetometer with Hall probes that works with the 3D printer principle was designed and tested to measure the magnetic field. Magnetic field changes were measured according to the surface coordinates on the measurement platform or measuring cell. Numerous experimental measurements of gradient magnetic fields generated by permanent magnets have been taken. The results obtained from the studies and results from the proposed empirical models were compared.
\end{abstract}

\section{INTRODUCTION}

Many and different kinds of mixtures, ranging in size from nanometers to several hundreds of micrometers, are used in chemical and biotechnological processes. Separation processes are commonly used to purify or extract these mixtures with various physicochemical properties according to some components [1]. Most mixtures used in chemical and biotechnological processes contain magnetic (para or ferro) particles [2]. The most obvious example of these particles is magnetotactic bacteria and red blood cells [3], [4], [5], [6], [7] Non-magnetic bioparticles that do not show magnetic properties can also be magnetized by adding artificial magnetic particles (magnetite, chromium, nickel, etc.) [4], [8]. Conventional (classical) separation systems are not sufficient to separate extremely small $(<1 \mu \mathrm{m})$ particles. Even though these particles are very small in size, they can be separated efficiently in magnetic separators [5], [6], [8], [9], [10], [11], [12]. Matrix elements of magnetic separators, especially ferromagnetic materials, can be easily magnetized using an external magnetic field. Through this applied magnetic field, high gradient magnetic fields are produced in the magnetized matrix regions (active regions) of magnetic separators [13],

(C) 2021 The Author(s). This is an open access article distributed under the terms of the Creative Commons Attribution License, which permits unrestricted use, distribution, and reproduction in any medium, provided the original author and source are credited. 
[14], [15] , [16]. Magnetic bioparticles can be easily separated by being exposed to the effect of great magnetic force if they are in these regions [9], [17], [18]. Gradient magnetic fields are also widely used in many areas of chemical engineering [19]. Advantages of gradient magnetic fields for intensification of heat and mass transfer processes that occur especially in catalytic reactors have been presented in the literature [20], [21], [22]. Furthermore, the effects of gradient magnetic fields on the acceleration of chemical processes on water and other chemical suspensions at the molecular level have also been presented in the literature [23], [24], [25], [26], [27], [28], [29].

The magnetic force acting on the magnetic bioparticles in the active regions of the separator is calculated as follows;

$$
\bar{F}_{m}=\frac{k_{p} w_{p}}{\mu_{0}} \nabla \frac{B^{2}}{2}
$$

Where, $k_{p}$ is the magnetic susceptibility of the particle, $\mu_{0}=4 \pi \times 10^{-7} \mathrm{H} / \mathrm{m}$ is the vacuum permeability, $w_{p}$ is the volume of the particle, and $B$ is external magnetic field intensity. Since this force $\left(\bar{F}_{m}\right)$ is many times greater than other forces between bioparticles (Van der Waals, electrostatic, drift, etc.), magnetic bioseparation can be performed effectively. In general, the magnetic field force determined by Equation (1) should be kept as high as possible for effective separation of bioparticles. As can be seen in Equation (1), the magnitude of the magnetic force affecting bioparticles is directly proportional to the magnetic field intensity $(B)$ and field gradient $(d B / d z)$. In bioseparation procedures, considering that the magnetic susceptibility level of particles is around $k_{p}=(0 \cdots 28,7) \times 10^{-5}[6]$ and magnetic field gradient is around $d B / d z=2,5 \times 10^{5} \mathrm{~T} / \mathrm{m}$, it is understood that the force acting on the $100 \mu \mathrm{m}$ particle in biologic systems is around $F_{m}=1,5 \times 10^{-4} N$ [1]. On the other hand, magnetic force at these levels may be insufficient for the separation of bioparticles with weak magnetic properties. In this case, it is possible to increase the strength of the magnetic system by increasing the magnetic field gradient through the creation of new structures. For this reason, an approximate empirical model of the magnetic field gradient in the working areas of magnetic separators need to be determined. These empirical formulas, should then be evaluated, compared, and corrected with empirical coefficients according to data obtained from magnetic separation experiments. In general, the generation and calculation of magnetic field gradient is one of the most important fundamental problems of the electromagnetic field theory. The results of many studies on this topic have been presented in the literature [11], [17], [30]. Different methods are used for the calculation of magnetic field gradients in Electromagnetic Field Theory [31]. However, the formulas obtained by methods based on the calculation of the scalar magnetic field potential, commonly used in classical electromagnetic field theory, are complicated and not appropriate for the practical calculations in separation practice [9], [11], [17], [32]. This prevents both the progression of biomagnetic separation theory and the expansion of its applicability in different areas. At the same time, the different geometries used in various new structures of the developed magnetic separators, cause new problems in the calculation and evaluation of magnetic field gradients. For that reason, new simple solution methods need to be developed to calculate gradient magnetic fields. For example, in recent years, gradient magnetic fields of $N d F e B$ or other types of permanent magnets, which have different geometric structures, have been used in nano and micro fluidic technological systems. However, the necessity of using simple methods to calculate gradient magnetic fields remains valid in such systems [33], [34].

In this study, two main problems in terms of both theoretical and practical were solved. First, a simple magnetic device or magnetometer was designed to determine the magnetic field with high precision of about 0,01 $\mathrm{mm}$. And then, empirical expressions were produced to determine the magnetic field or field gradients. For this purpose, using the $3 D$ printer principle, a magnetometer with Hall probes was designed and used. This device can measure the magnetic field in three dimensions $(x, y, z)$ automatically at precise intervals $(<0,1 \mathrm{~mm})$. In the experiments, the gradient magnetic fields generated by rectangular and disc-shaped $\mathrm{NdFeB}$ type magnets even electromagnets were measured. At the same time, empirical models were created according to the experimental results. The measurement results were demonstrated with tables and graphics, and necessary comparisons, and recommendations have been presented. 


\section{MATERIAL AND METHOD}

Numerous methods and setups are used to measure the gradient magnetic fields that generated in narrow air gaps in magnetic systems [11], [17], [35]. The most practical and suitable of these methods is the use of Hall probes [36]. Magnetic field intensity $(|\boldsymbol{B}|)$ of uniform and symmetrical magnetic fields in a small air gaps and the gradient of this field $(\nabla|\boldsymbol{B}|)$ can be easily measured with Hall probes [36]. However, there are many challenges in measuring $|\boldsymbol{B}|$ or $\nabla|\boldsymbol{B}|$ between poles with different geometries (sphere, cylinder, prism, etc.) because this magnetic fields are strongly non-uniform fields. Therefore, in biochemical separation process, they show different magnetic field effects at different points of the working regions. Foremost among these challenges, is the safe fixed of the Hall probe in the measurement area and the precise selection of measurement ranges. For these non-symmetrical air gap geometries, it is especially important that the Hall probe is safely fixed at different points for protected from vibrations and oscillations, and measurement intervals can be adjusted automatically. Because of the errors caused by failure to meet these conditions affect the measurement results negatively. Developing new methods to eliminate such problems has always been in the center of attention of researchers [36], [37]. In this study, we described the details of the method to eliminate such problems.

\subsection{MATERIAL}

\subsubsection{D PRINTER PRINCIPLES MAGNETOMETER WITH HALL PROBES}

The magnetometer, which is an automatic measurement device that uses the Hall probe and works with the $3 D$ printer principle, has been designed and tested to measure gradient magnetic fields in different air gaps in magnetic systems [38]. This magnetometer shown in Figure 1a has a programmable feature. Moreover, the Hall probe placed in the air gap between the magnets can move independently from each other in the $x, y, z$ directions. The Hall probe which is fixed, can be moved directed independently in each direction with using three step motors. The working area of the magnetometer, designed by us produced in the TEKNOPARK that is part of Inonu University, is $30 \times 20 \times 24 \mathrm{~cm}$, and its overall size is $48 \times 50 \times 40 \mathrm{~cm}$.
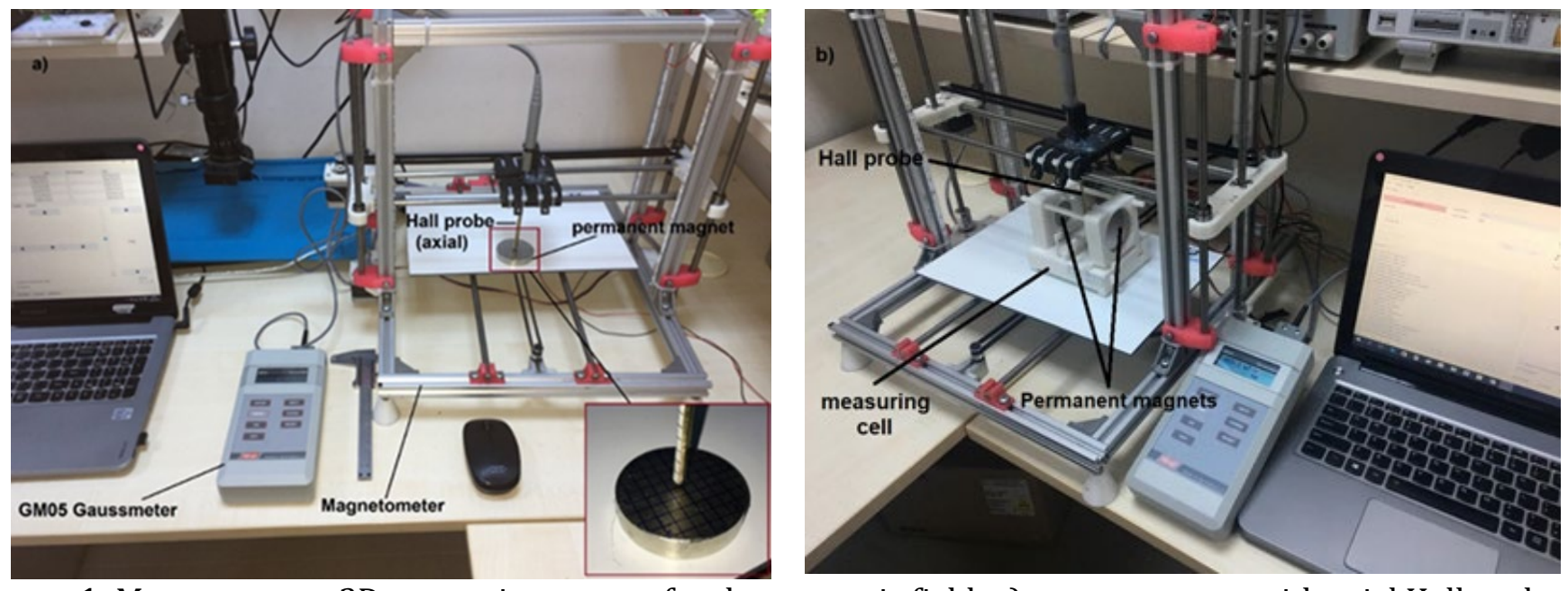

Figure 1: Magnetometer,3D measuring system for the magnetic field; a) magnetometer with axial Hall probe on measuring platform, b) magnetometer with measuring cell.

The magnetometer's control system is supplied by a 12V DC voltage source. The magnets can be placed on the platform shown in Figure 1a or measurement cell shown in Figure 1b. Measuring cell has various apparatus for different magnets. Due to the special design of the measuring cell, magnets with different geometries can be placed in, and the distance between these magnets can be adjusted (Figure 1b). The measuring cell also provides the measurement of magnetic fields in small size solenoid and electromagnet. By modifying / configuring the unit on which the Hall probe is attached for fixation, it is also possible to determine the magnetic susceptibility of smallsized magnetic materials or mixtures. 


\subsubsection{DESCRIPTION OF THE MATERIAL}

In the experiments, $N d F e B$ type permanent magnets that have high magnetic energy $\left(\left(320 \mathrm{~kJ} / \mathrm{m}^{3}\right)\right.$ and most preferred in High Gradient Magnetic Separator (HGMS) and Magnetic Drug Targeting (MDT) systems were used. Samples are rectangular $N d F e B$ magnet $30 \times 10 \times 5 \mathrm{~mm}(2 a \times 2 b x l)$ dimensions and disc-shaped magnets with diameter $d=50 \mathrm{~mm}$, thickness $l=10 \mathrm{~mm}$ (Figure 2). Magnetic field intensity on the surface of the magnets has been measured as $B_{0}=250 \mathrm{mT}$ and $B_{0}=300 \mathrm{mT}$, respectively. Despite these results, in the designed magnetometer, it is possible to easily measure the magnetic fields created by magnets containing $A L N I C O, S m C o_{5}$ type, and new type of hexaferrites and composites based on them [39] used in magnetic systems. In the magnetometer, it is possible to measure the fields of solenoids and similar winding types of magnetic field sources with precision.

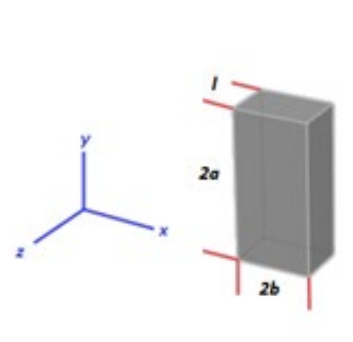

a)

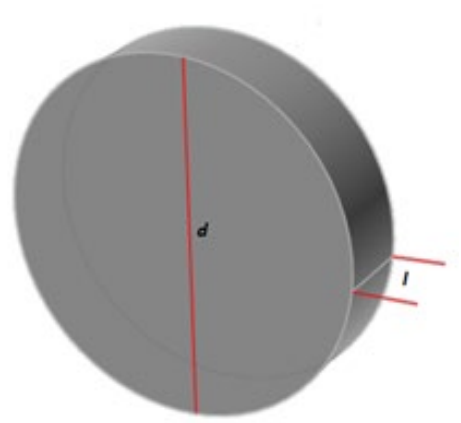

b)

Figure 2: a) Rectangular and b) Disc-shaped NdFeB magnets.

\subsubsection{MEASUREMENT SYSTEM}

GM05 type Gaussmeter with Hall probe (HIRST-Magnetic Instrument Ltd-UK) was used in the experiments (Figure 3). GM05 Gaussmeter has measurement and evaluation features of AC magnetic fields in DC and $15 \mathrm{~Hz}-$ $10 \mathrm{kHz}$ frequencies. Gaussmeter's operating temperature is $0^{\circ} \mathrm{C} \mp 50^{\circ} \mathrm{C}$, and reproducibility is $5 \%$. GM05 has two Hall probes, $l=90 \mathrm{~mm}$ long, 90x0,6x4 $\mathrm{mm}$ transverse, and $5 \mathrm{~mm}$ diameter axial (Figure 3). These probes with an extension connection cable RS232 enables the measuring device to be kept far enough from the magnetic field source and to protect the device from external electromagnetic effects. When necessary, the magnetometer can be protected from the influence of external magnetic fields with an outer shield such as a multilayered $\mathrm{Ni}_{80} \mathrm{Fe}_{20} / \mathrm{Cu}$ film [40]. In principle, all similar Gaussmeters with Hall probes, like the measuring device, can also be used in this system. Besides, measurement results can be obtained more easily with the USB and RS232 connections in the new GM07 / GM08 Gaussmeters with GM modifications. The devices' software is also Windows compatible.

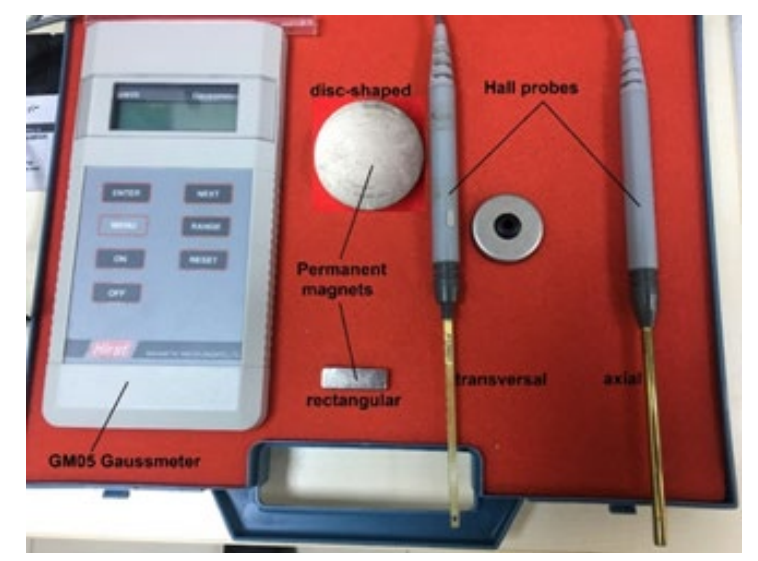

Figure 3: Magnetic field measuring elements. GM05 type Gaussmeter, Hall probes (axial and transversal) and permanent magnets. 
Hatice Bilgili, Teymuraz Abbasov, and Yusuf Baran

\subsection{THE METHOD}

The measuring points in the air gap are automatically selected according to the preloaded program, and the measured values are recorded at the exact steady state of the Hall probe. These measurement results can be recorded on the computer connected to the magnetometer and displayed in tabular or graphical format.

\subsubsection{THE MATHEMATICAL FORMULATION OF THE PROBLEM}

The theory and practice of magnetic separation of bioparticles, especially MDT, are generally like HGMS processes. Despite this, both MDT and biomagnetic separation of different particles have many different properties. Unlike HGMS, magnetic bioseparation or MDT have the following specific characteristics:

Gradient magnetic fields are mainly generated by permanent magnets. Permanent magnets can have different geometries (square, rectangle, cylindrical, disc, etc.) and different dimensions.

In the areas of separation or drug targeting, the maximum value of magnetic field should be focused. For example, a cylindrical magnet should at least create $H>10^{5} \mathrm{~A} / \mathrm{m}$ magnetic field in the sick areas $(>5 \mathrm{~cm})$. On the other hand, in some experiments, it was determined that this distance would approximately be $<13 \mathrm{~cm}$ in order to keep $0.5-5.0 \mathrm{~nm}$ sized iron-carbon particles inside the body [41]. Therefore, the generated magnetic field force should have higher values in all points of working regions.

In the MDT, the size of drug carrier particles is desired to be $<5-6 \mu \mathrm{m}$. If the particles are larger than these sizes, cause blockages in capillaries [42]. Therefore, it is desired that the magnetically drug-attached carrier particles have a smaller size. On the other hand, reduction in particle size causes the effect of the magnetic field on these particles to weaken and the required blocking time in the affected sick areas increases. While blocking time is $<$ $150 \mathrm{~s}$ in the field of $B=0.35 \mathrm{~T}$ area, blocking time can be $<450 \mathrm{~s}$ in the field of $B=0.1 \mathrm{~T}$ area [42].

The particles in bioseparation procedures could have solid, liquid, deformable plastic, and other different physicochemical properties. On the other hand, suspensions, emulsions, plasma, or highly concentrated medias used in magnetic separation processes in chemical, medical, and biological environments may have rheological properties. Therefore, it is necessary to consider the effect of these properties of the carrier media on the separation process.

The size of the magnets or sources of electromagnetic fields used in magnetic bioseparation processes must be small, and the magnetic fields generated must be large enough. Moreover, it is obvious that the energy and the magnetic field intensity induced by permanent magnets are limited. Therefore, it is necessary to know the changes of the magnetic field intensity $B$ and gradient $\nabla B$ in the target region of the magnetic field for each separation process. This can be obtained from basic empirical and theoretical models that are based on many experimental results for each state. There are many theoretical models for the variation of the magnetic field intensity generated by permanent magnets [43]. In micro and nano fluidic systems in biotechnology, rectangular, disc and cylindrical shaped magnets are commonly preferred [33], [43]. The characteristic dimensions of rectangular and disc-shaped magnets are shown in Figure 2.

In terms of practical application for bioseparation and drug targeting systems, the component of the magnetic field on the axis perpendicular to the magnet surface (e.g., z axis in Figure 2) is more important. In general, the determination of all components of the magnetic field generated by magnets in practical engineering calculations is modeled by numerical methods [33], [41], [42]. In addition, approximate and useful formulas for magnetic field calculation of magnets are also presented in the literature [33], [43]. Some of these formulas are given below. If $B_{0}$ is the magnetic field intensity at the center of magnet $(x=y=z=0), B_{z}$ component can be determined as follows.

for rectangular magnets [43];

$$
B_{z}=\frac{B_{0}}{\left[1+4(z / d)^{2}\right]^{3 / 2}}
$$

or disc-shaped magnets [43];

$$
B_{Z}=\frac{B_{0} b^{2}}{\sqrt{a^{2}+b^{2}}\left(a^{2}+b^{2}+z^{2}\right)^{3 / 2}}\left[\frac{1}{a^{2}+z^{2}}+\frac{1}{b^{2}+z^{2}}\right]
$$

International Journal of Engineering Science Technologies 
for cylindrical magnets [41];

$$
B_{z}=\frac{c_{1}}{(z / d)^{2}}
$$

for magnetic circuit with parabolic shape confocal pole (MCPSP) [41];

$$
B_{z}=\frac{c_{2}}{(z / b)^{f}}
$$

Here $C_{1}$ and $C_{2}$ are constants, $C_{1}=5 \pi \cdot 10^{-2} T, C_{2}=30,4 \cdot \pi \cdot 10^{-2} T, f=0,67[41]$.

Although Equation (2) and Equation (3), contain simple expressions, they may not be suitable for theoretical investigations. Equation (4) and Equation (5) show that the magnetic field intensity on the surface of the magnets $(z=0)$ is infinite. This is incompatible with reality for real magnetization systems. To simplify / eliminate these issues, in this study, it is proposed to model the change of magnetic field intensity on the $z$-axis with the following two approximate formulas.

$$
\begin{aligned}
& B_{z}=B_{0} e^{\left[-k_{1}\left(\frac{z}{d}\right)^{m}\right]} \\
& B_{z}=\frac{B_{0}}{1+k_{2}\left(\frac{z}{d}\right)^{2}}
\end{aligned}
$$

Here, $m, k_{1}$ and $k_{2}$ are experimentally determined correction coefficients. According to the results of the experiments, the coefficients $m, k_{1}$ and $k_{2}$ included in Equation (6) and Equation (7) can be determined for each magnet geometry. With the approach in this study, the compatibility of the experimental measurements made in the $z$ axis direction of rectangular and disc-shaped $N d F e B$ magnets to the proposed models were evaluated.

\section{RESULT AND DISCUSSION}

In the experiments, $|B|$ was measured according to surface coordinates $(x, y, z)$ of the magnet using the field generated by the magnets fixed on the measuring platform. In Figure 4, magnetic field measurements were made on the surface of the rectangular shaped magnet in $x$ axis symmetry $(x=3 \mathrm{~cm}, y=$ constant, $z=0)$. At all levels $(y=$ 0 and $y= \pm 4 \mathrm{~mm}$ ), it has been observed that the magnetic field values on the $x$ axis are symmetrical. An increase in magnetic field intensity was observed at the edges of the magnet [43]. In the experiments, 1178 measurements were made for different regions on the magnets. The results obtained are shown in Figure 5, Figure 6, and Figure 7.

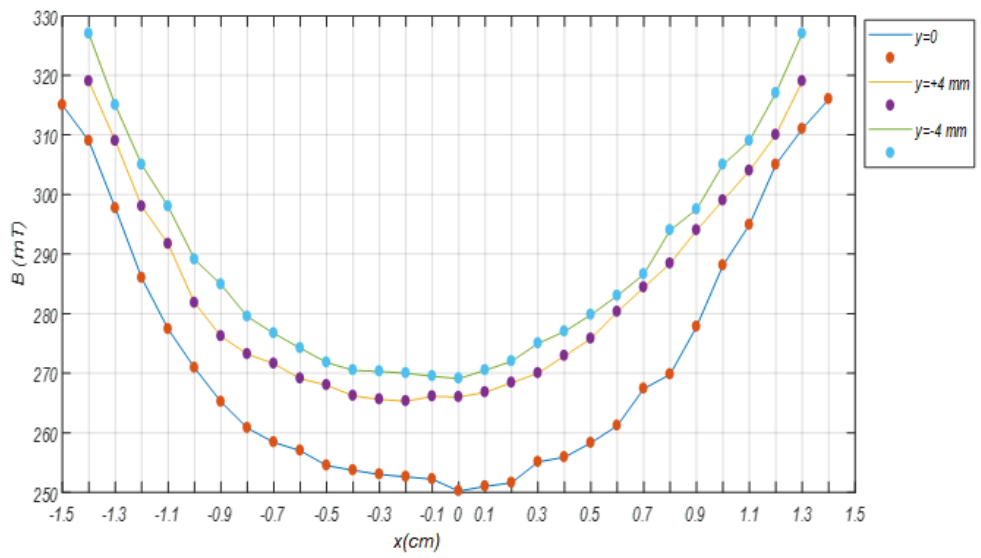

Figure 4: Magnetic field measurement results at the surface of the rectangular magnet with respect to the symmetry of the $x$ axis. 
In the practical applications of biotechnological systems, the magnetic force acting on bioparticles, which are generally separated, is at a specific distance from the magnet surface. Therefore, from the results obtained from measurements, the direction of $z$ axis was evaluated.
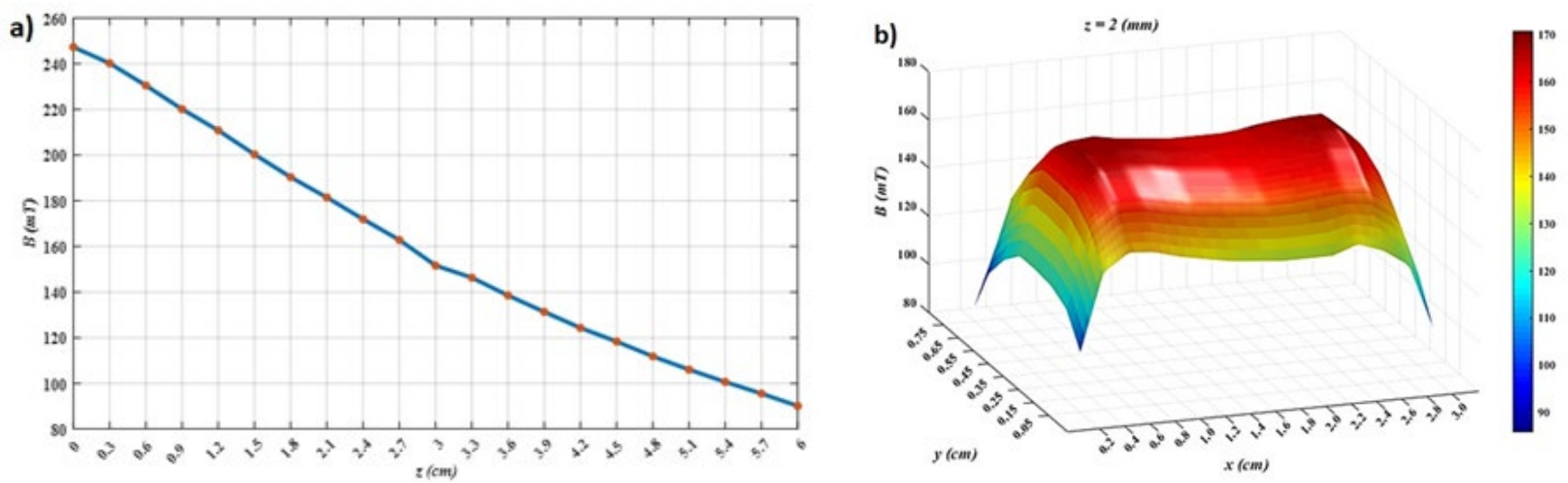

Figure 5: Magnetic field intensity changes of rectangular $N d F e B$ magnet; a) from the center of the magnet in the direction of the $z$ axis, b) $z=2 \mathrm{~mm}$ in the $x$ and $y$ direction at the.

Figure 5 shows the graph of the magnetic field intensity changing as it moves away from the center of the rectangular $N d F e B$ magnet ( $10 \times 5 \times 30 \mathrm{~mm}$ dimensions) in the direction of the $z$ axis. As can be seen in Figure $5 \mathrm{a}$, the magnetic field intensity decreases rapidly as it moves away from the surface of the magnet. This reduction becomes faster the further away from the surface. However, it is seen that the decrease in the magnetic field is still at a certain level, even at a sufficient distance from the magnet surface $(z=6 \mathrm{~cm})$. At this distance, the magnetic field has decreased from $B=250 \mathrm{mT}$ to around $B=90 \mathrm{mT}$, that is, it has decreased by approximately $64 \%$. At $z=3 \mathrm{~cm}$ from the surface, this change is around $40 \%$. Similar variation is clearly seen in the $x$ and $y$ directions when $z=$ constant (Figure $5 \mathrm{~b}$ ). Unlike, the magnetic field of the disc-shaped $N d F e B$ magnet decreases faster as it moves away from the center in the $z$ axis direction. This change is shown in Figure 6 and Figure 7.

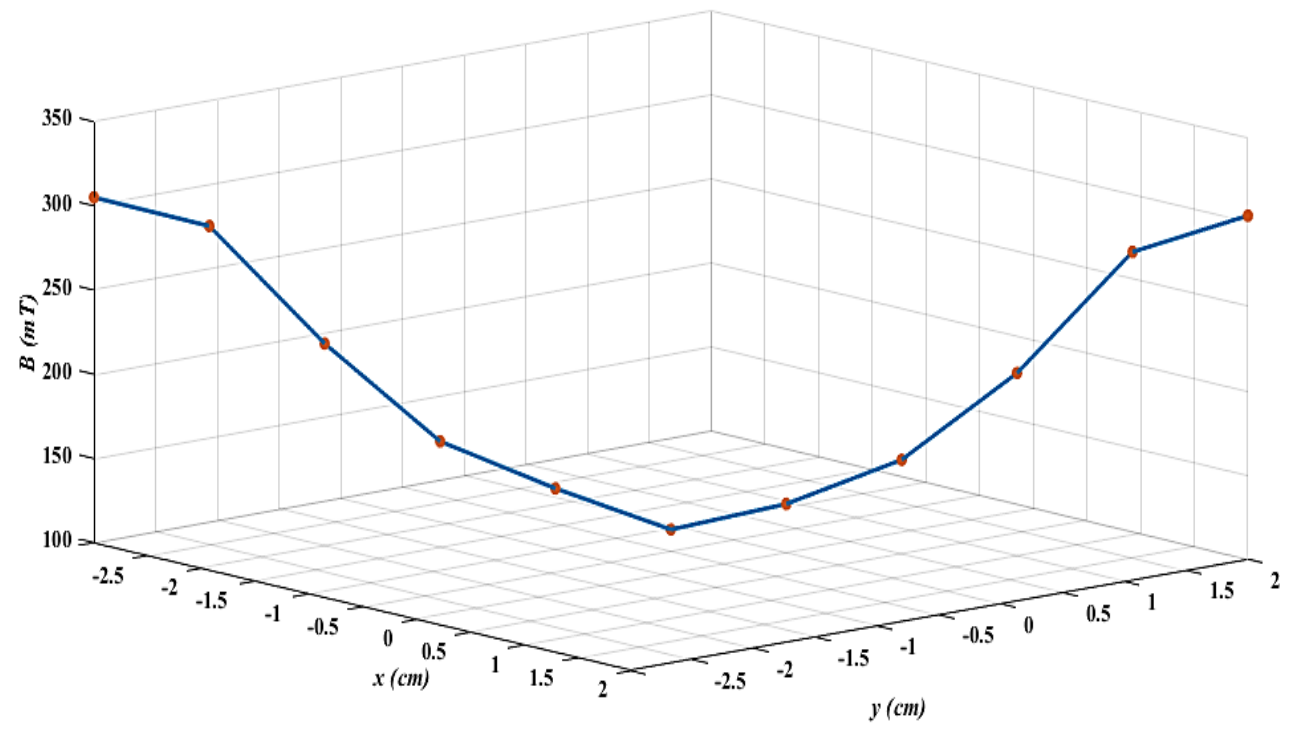

Figure 6: The graph of change in $B(x, y)$ on the surface $(z=0)$ of the disc-shaped $N d F e B$ magnet.

Figure 6 shows the measurement results of the changes in the $x$ and $y$ directions of the magnetic field intensity on the surface of the disc-shaped $N d F e B$ magnet $(z=0)$. As seen in Figure 6 , the magnetic field intensity has a clear symmetry. However, the further away from the center of the disc $(0,0,0)$, this field intensity gets greater. These similar change characteristics for permanent magnets are also sufficiently explained in the literature [43]. 

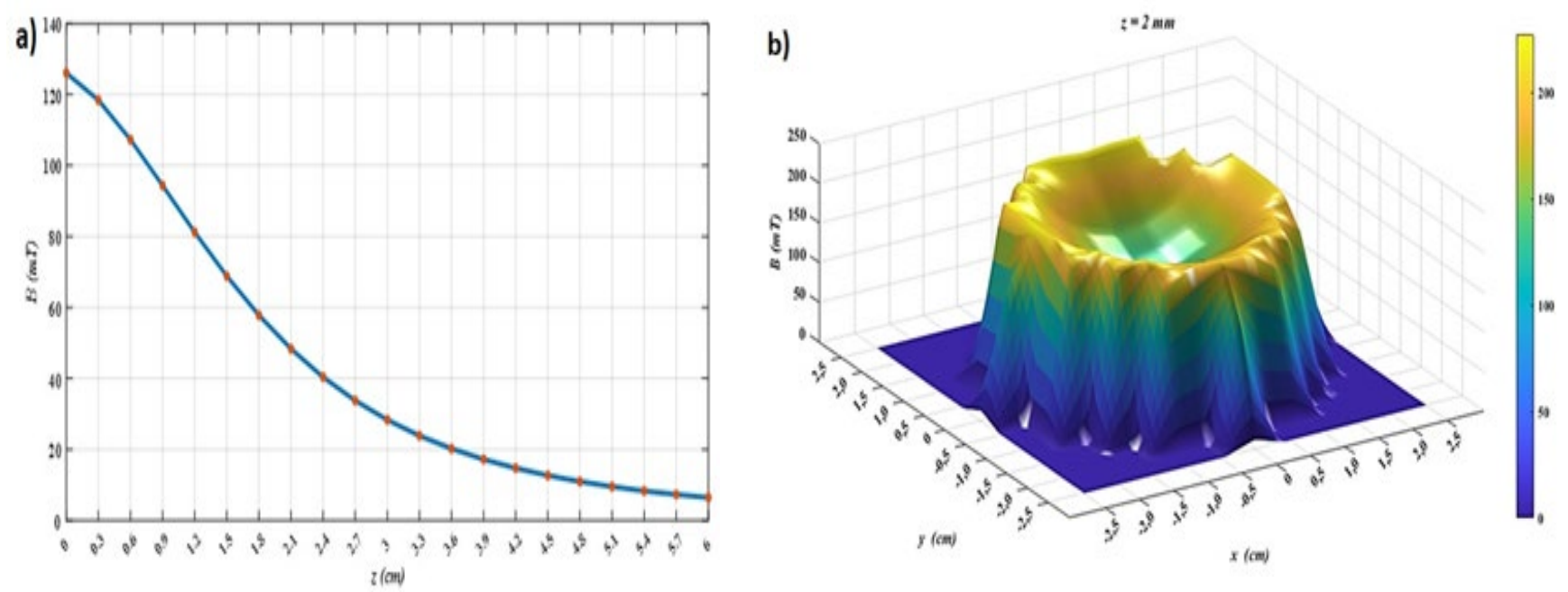

Figure 7: Magnetic field intensity changes of disc-shaped $N d F e B$ magnet; a) $B(z)$ graph from the center of the magnet in the direction of the $z$ axis, b) $B(x, y)$ graph at the $z=2 \mathrm{~mm}$.

As seen in Figure 7a, the magnetic field intensity decreased from $B=130 \mathrm{mT}$ to around $B=34 \mathrm{mT}$ that is around $74 \%$ at a distance $z=3 \mathrm{~cm}$ from the surface of the magnet. This drop rate in the disc-shaped magnet is approximately twice the drop rate of the rectangular magnet. Similar changes of the magnetic field on the surfaces of magnets have been observed on other coordinates (Figure $7 \mathrm{~b}$ ).

In general, these changes are of great importance in bioseparation processes in the gradient magnetic field. Consideration of this phenomenon is critical especially in in-Vivo and in-Vitro applications of MDT systems. Because the effectiveness of the MDT technique is determined by the level of the magnetic field or field gradient applied to magnetic drugs [33]. As a result of these evaluations, it is clearly seen that it is more advantageous to use rectangular shaped magnets in magnetic separation processes. However, the effect of magnetic field gradient may be more dominant for targeting magnetic drugs in regions close to the surface of the magnet. In this case, it would be more advantageous to use disc-shaped magnets. The magnetic field for each system must be examined separately if magnets with different shapes are used in biomagnetic separation and drug targeting systems. In other words, in systems, both intensity and gradient change should be evaluated for the magnetic field at different points of the working area by measuring. The three-dimensional magnetic field measuring device, we covered in our study, is particularly important in this respect.

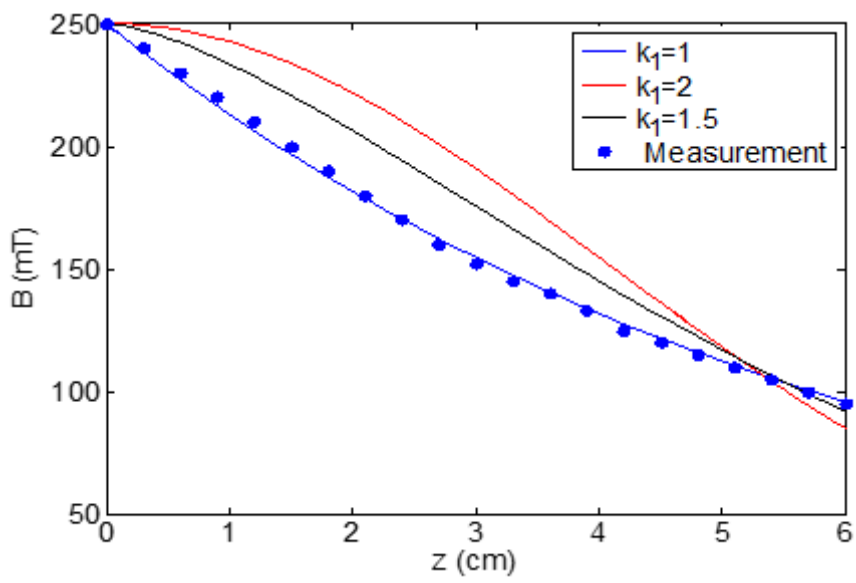

Figure 8: The variation of the magnetic field intensity along $z$ axis for rectangular shaped magnets.

In Figure 8 and Figure 9, the results of both experiments and calculations made according to Equations (6) and (7) are given together for comparison. In Figure 8, according to the formula in Equation (6), the changes of the magnetic field intensity in the $z$-direction at different values of the correction coefficient $\left(k_{1}\right)$ are shown. As shown in Figure 8, the measurement data and the data obtained from Equation (6) are similar in nature for $m=1$. However, 
Equation (6) does not conformity with the results of the experiments at $m>1$ values. Also, as $m$ increases, the unconformity increases rapidly. Therefore, Equation (6) gives more accurate results for rectangular magnets only at $m=1$.

However, these graphics do not match in terms of the values. Although the change of the magnetic field with the exponent law according to the $z$ distance is theoretically convenient, both the field intensity and the field gradient values calculated according to Equation (6) are obtained with errors. According to this result, it is not advantageous to use the magnetic field gradient generated by rectangular magnets in magnetic separators.

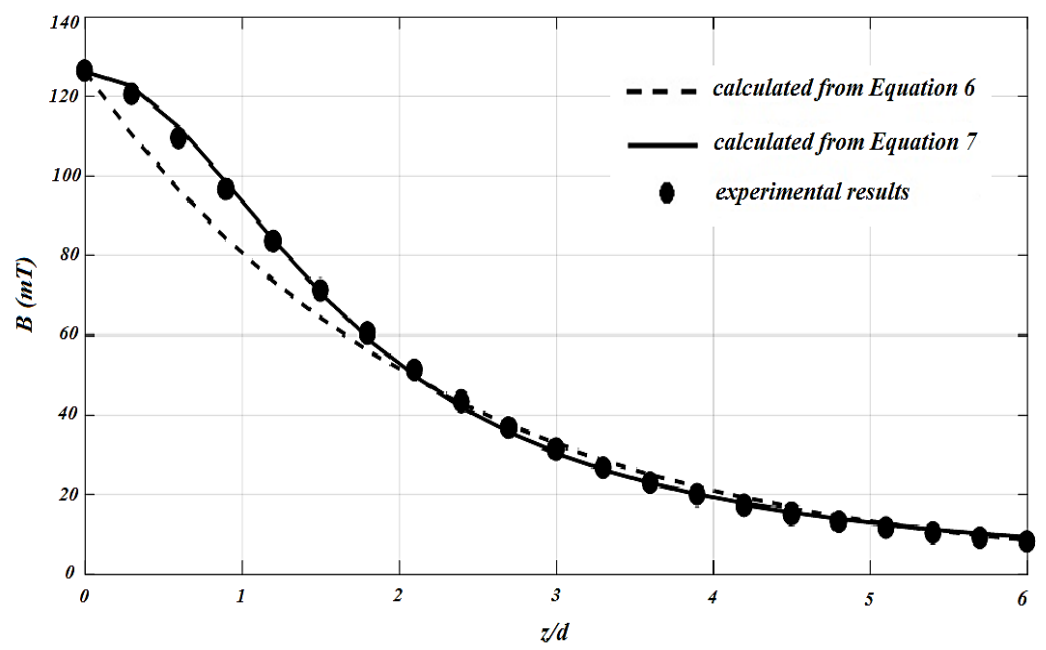

Figure 9: Variation of the magnetic field intensity along z axis for disc-shaped magnets.

Alternatively, it is clear from Figure 9 that the empirical formula proposed in Equation (7) fits better with experimental data for disc-shaped magnets. The variation of the magnetic field intensity in the $z$ axis direction of the disc-shaped magnet theoretically and experimentally coincides with each other. Moreover, the correction coefficient $k$ included in Equation (7) can be determined by making fewer number (2 or 3) of experimental measurements for magnets with different sizes. This clearly shows that the theoretical model given in Equation (7) is more advantageous for practical calculations. In fact, the performance of biomagnetic separators are determined by the magnetic field gradient rather than magnetic field intensity. Given this situation, $|B \nabla B|$, which is obtained from Equation (6) and Equation (7) and defined as the force factor, is also useful to evaluate. Figure 10 shows the graph of the force factor change in the $z$ direction. As can be seen in Figure 10, under equal conditions, the force factor $(|B \nabla B|)$ obtained from Equation (7) seems to be more effective. It is more advantageous to use the theoretical model determined by Equation (7) to evaluate the performance of these separators during the design and use of biomagnetic separators.

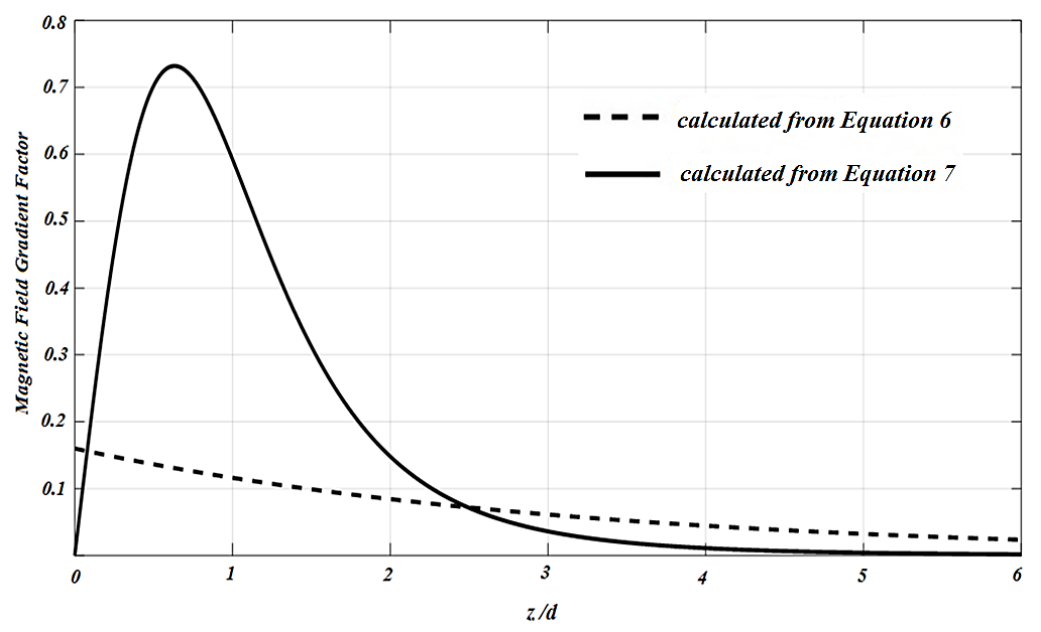

Figure 10: The variation of the dimensionless magnetic field gradient factor along the $z$ axis International Journal of Engineering Science Technologies 


\section{CONCLUSIONS}

The following results are obtained from the studies conducted:

- The use of gradient magnetic fields remains an important method in developing and using new technologies.

- To use gradient magnetic fields effectively in different systems, the main magnetization system (magnet, coil, electromagnetic, etc.) should be professionally designed and selected. The magnetic field intensity and gradient of the magnetic system must be measured and evaluated for each structure. In this case, some classical methods may not be enough. Therefore, simpler magnetometers should be designed or created for new structures, especially for smaller or narrower working regions.

- Permanent magnet or magnetizing elements used in these systems may exhibit different performance depending on the structure of systems, the geometry of the working area and the characteristics of the environment used. Measuring and modeling the magnetic field and field gradient is required to correctly evaluate these properties both theoretically and experimentally.

- To evaluate the changes of the magnetic field and the field gradient, $3 D$ printer principle designed measurement setup (magnetometer) with Hall probes can be used. This magnetometer, which makes accurate measurements in three directions, allows to adjust the measurement steps less than $<0,1 \mathrm{~mm}$ and to obtain the measurement data both in tabular and graphical formats.

- The specially designed measuring cell assembly can evaluate measuring magnetic field values at different points of magnetic elements of different dimensions and geometries.

- Designed magnetometer can be used to evaluate the magnetic field and gradient in special magnetic separators for chemical and biomedical engineering processes.

- The $3 D$ magnetic field measurement device, with Hall probe can be used with the same success to measure and evaluate the magnetic fields in different magnetophoresis processes for nano and micro fluidic systems.

- The simple empirical formula shown in Equations (6) and (7) can be used to evaluate the experimental results, magnetic field intensity, and magnetic field gradient.

- The proposed methods will significantly contribute to the theoretical and experimental determination of the performance characteristics of magnetophoresis / magnetic separation processes, which have wide application areas.

\section{SOURCES OF FUNDING}

This research received no specific grant from any funding agency in the public, commercial, or not-for-profit sectors.

\section{CONFLICT OF INTEREST}

The author have declared that no competing interests exist.

\section{ACKNOWLEDGMENT}

This study was supported by Inonu University Scientific Research Projects Coordination Unit (BAP) within the scope of the project numbered FDK-2017-775.

\section{REFERENCES}

[1] P. Van Hee, M. Hoeben, R. Van der Lans, and L. Van der Wielen, "Strategy for selection of methods for separation of bioparticles from particle mixtures," Biotechnology and Bioengineering, vol. 94, no. 4, pp. 689709, 2006.2 [Online]. Available: https://onlinelibrary.wiley.com/doi/pdfdirect/10.1002/bit.20885?download=true.

[2] A. L. Elrefai, T. Yoshida, and K. Enpuku, "Magnetic parameters evaluation of magnetic nanoparticles for use in biomedical applications," Journal of Magnetism and Magnetic Materials, vol. 474, pp. 522-527, 2019. 
[3] R. E. Dunin-Borkowski, M. R. McCartney, R. B. Frankel, D. A. Bazylinski, M. Pósfai, and P. R. Buseck, "Magnetic microstructure of magnetotactic bacteria by electron holography," Science, vol. 282, no. 5395, pp. 18681870, 1998. [Online]. Available: https://science.sciencemag.org/content/sci/282/5395/1868.full.pdf.

[4] U. Lins and M. Farina, "Phosphorus-rich granules in uncultured magnetotactic bacteria," FEMS microbiology letters, vol. 172, no. 1, pp. 23-28, 1999.

[5] D. Melville, F. Paul, and S. Roath, "Direct magnetic separation of red cells from whole blood," Nature, vol. 255, no. 5511, pp. 706-706, 1975. [Online]. Available: https://www.nature.com/articles/255706a0.pdf.

[6] A. Bahaj, J. Watson, and D. Ellwood, "Determination of magnetic susceptibility of loaded micro-organisms in bio-magnetic separation," IEEE Transactions on Magnetics, vol. 25, no. 5, pp. 3809-3811, 1989.

[7] A. Bahaj, P. James, and F. Moeschler, "Efficiency enhancements through the use of magnetic field gradient in orientation magnetic separation for the removal of pollutants by magnetotactic bacteria," Separation science and technology, vol. 37, no. 16, pp. 3661-3671, 2002.

[8] C. T. Yavuz, A. Prakash, J. Mayo, and V. L. Colvin, "Magnetic separations: from steel plants to biotechnology," Chemical Engineering Science, vol. 64, no. 10, pp. 2510-2521, 2009.

[9] M. Zborowski and J. J. Chalmers, Magnetic cell separation. Elsevier, 2011.

[10] C. Hoffmann, M. Franzreb, and W. Holl, "A novel high-gradient magnetic separator (HGMS) design for biotech applications," IEEE transactions on applied superconductivity, vol. 12, no. 1, pp. 963-966, 2002.

[11] V. Karmazin, "Theoretical assessment of technological potential of magnetic and electrical separation," Magnetic and Electrical Separation, vol. 8, 1970.

[12] V. Schaller et al., "Motion of nanometer sized magnetic particles in a magnetic field gradient," Journal of Applied Physics, vol. 104, no. 9, p. 093918, 2008.

[13] J. Zeng, X. Tong, F. Yi, and L. Chen, "Selective capture of magnetic wires to particles in high gradient magnetic separation," Minerals, vol. 9, no. 9, p. 509, 2019.

[14] H. Kang, J. Kim, H. Cho, and K.-H. Han, "Evaluation of Positive and Negative Methods for Isolation of Circulating Tumor Cells by Lateral Magnetophoresis," Micromachines, vol. 10, no. 6, p. 386, 2019.

[15] J. Lunacek et al., "Efficiency of high gradient magnetic separation applied to micrometric magnetic particles," Separation Science and Technology, vol. 50, no. 16, pp. 2606-2615, 2015.

[16] Z. Yildiz, T. Abbasov, and A. Sarimeseli, "Effect of the Magnetization Properties of the Granular Beds and the Operating Parameters on the Removal Ferrous Particles From the Waters by Using Magnetic Filter," Particulate Science and Technology, vol. 31, no. 2, pp. 109-113, 2013.

[17] J. Svoboda, Magnetic techniques for the treatment of materials. Springer Science \& Business Media, 2004.

[18] A. Khaligh and H. B. Ghavifekr, "Design of a MEMS-based magnetophoresis micro-separator," in 201624 th Iranian Conference on Electrical Engineering (ICEE), 2016: IEEE, pp. 1495-1498.

[19] P. Hajiani and F. Larachi, "Ferrofluid applications in chemical engineering," Int. Rev. Chem. Eng., vol. 1, pp. 221-237, 2009.

[20] M. Munteanu and F. Larachi, "Inhomogeneous magnetic field effects on the hydrodynamic properties of multiphase catalytic reactors," International Review of Chemical Engineering, vol. 2, no. 1, pp. 150-154, 2010.

[21] M. Rolland, F. Larachi, and P. Hajiani, "Axial dispersion in nanofluid Poiseuille flows stirred by magnetic nanoagitators," Industrial \& Engineering Chemistry Research, vol. 53, no. 14, pp. 6204-6210, 2014.

[22] F. Larachi, "Experimental and theoretical exploration of weak-and strong-gradient magnetic fields in chemical multiphase processes," Modeling of process intensification. Weinheim/Germany: Wiley-VCH, 2007.

[23] V. Zablotskii, T. Polyakova, O. Lunov, and A. Dejneka, "How a high-gradient magnetic field could affect cell life," Scientific reports, vol. 6, no. 1, pp. 1-13, 2016.

[24] L. Mulay and I. L. Mulay, "Magnetic susceptibility: instrumentation and analytical applications including bioscience, catalysis, and amorphous materials," Analytical Chemistry, vol. 52, no. 5, pp. 199-214, 1980.

[25] C. L. Hill, A. Lamotte, W. Althoff, J.-C. Brunie, and G. M. Whitesides, "High-gradient magnetic filtration of small particles of ferro-, ferri-, and paramagnetic catalysts and catalyst supports," Journal of Catalysis, vol. 43, no. 1-3, pp. 53-60, 1976.

[26] S. Ge et al., "Magnetic levitation in chemistry, materials science, and biochemistry," Angewandte Chemie International Edition, vol. 59, no. 41, pp. 17810-17855, 2020.

[27] C. A. Sobecki, J. Zhang, and C. Wang, "Dynamics of a Pair of Paramagnetic Janus Particles under a Uniform Magnetic Field and Simple Shear Flow," Magnetochemistry, vol. 7, no. 1, 2021. 
[28] J. Zhang and C. Wang, "Numerical study of lateral migration of elliptical magnetic microparticles in microchannels in uniform magnetic fields," Magnetochemistry, vol. 4, no. 1, p. 16, 2018.

[29] A. Eisenträger, D. Vella, and I. M. Griffiths, "Particle Capture efficiency in a multi-wire model for high gradient magnetic separation," Applied Physics Letters, vol. 105, no. 3, p. 033508, 2014.

[30] W. H. Hayt Jr, J. A. Buck, and M. J. Akhtar, Engineering Electromagnetics| (SIE). McGraw-Hill Education, 2020.

[31] G. Lehner, Electromagnetic field theory for engineers and physicists. Springer Science \& Business Media, 2010.

[32] X. Zheng, Z. Xue, Y. Wang, G. Zhu, D. Lu, and X. Li, "Modeling of particle capture in high gradient magnetic separation: A review," Powder technology, vol. 352, pp. 159-169, 2019.

[33] E. P. Furlani, Permanent magnet and electromechanical devices: materials, analysis, and applications. Academic press, 2001.

[34] K. M. Krishnan, Fundamentals and applications of magnetic materials. Oxford University Press, 2016.

[35] S. Baik, D. Ha, R. Ko, and J. Kwon, "Magnetic field analysis of high gradient magnetic separator via finite element analysis," Physica C: Superconductivity, vol. 480, pp. 111-117, 2012.

[36] J. Sammer, M. Hubmann, F. Schauer, J. Schmidt, and J. Gerhold, "Automatic measurement of nonuniform magnetic fields with industrial Hall probes," IEEE transactions on magnetics, vol. 26, no. 5, pp. 2064-2066, 1990.

[37] S. A. Khashan, Y. Haik, and E. Elnajjar, "CFD simulation for biomagnetic separation involving dilute suspensions," The Canadian Journal of Chemical Engineering, vol. 90, no. 6, pp. 1450-1456, 2012.

[38] H. Bilgili, T. Abbasov, and Y. Baran, "Analysis and Experimental Tests of Gradient Magnetic Fields for Separation of Bioparticles," in II. International Battalgazi Multidisciplinary Studies Congress, Malatya, Turkey, M. Talas, Ed., 15-17 March 2019 2019: IKSAD, 2019, pp. 150-156.

[39] Y. Yang et al., "Influence of Nd-NbZn co-substitution on structural, spectral and magnetic properties of Mtype calcium-strontium hexaferrites Ca0. 4Sr0. 6-xNdxFe12. 0-x (Nb0.5Zn0. 5) x019," Journal of Alloys and Compounds, vol. 765, pp. 616-623, 2018.

[40] A. Trukhanov, S. Grabchikov, A. Solobai, D. Tishkevich, S. Trukhanov, and E. Trukhanova, "AC and DCshielding properties for the Ni80Fe20/Cu film structures," Journal of Magnetism and Magnetic Materials, vol. 443, pp. 142-148, 2017.

[41] O. Rotariu and N. J. Strachan, "Modelling magnetic carrier particle targeting in the tumor microvasculature for cancer treatment," Journal of magnetism and magnetic materials, vol. 293, no. 1, pp. 639-646, 2005.

[42] L. E. Udrea, N. J. Strachan, V. Bădescu, and O. Rotariu, "An in vitro study of magnetic particle targeting in small blood vessels," Physics in Medicine \& Biology, vol. 51, no. 19, p. 4869, 2006.

[43] Y. M. Pyatin, "Permanent magnets," Energiya, Moscow, 1980. 